\title{
Erratum: Systemic Characterization of Novel Immune Cell Phenotypes in Recurrent Pregnancy Loss
}

\author{
Frontiers Production Office ${ }^{*}$ \\ Frontiers Media SA, Lausanne, Switzerland
}

Approved by:

Frontiers Editorial Office,

Frontiers Media SA, Switzerland

*Correspondence: Frontiers Production Office production.office@frontiersin.org

Specialty section: This article was submitted to Immunological Tolerance and Regulation,

a section of the journal

Frontiers in Immunology

Received: 09 June 2021 Accepted: 09 June 2021

Published: 23 June 2021

Citation:

\section{An Erratum on}

Systemic Characterization of Novel Immune Cell Phenotypes in Recurrent Pregnancy Loss By Liu H, Lin X-X, Huang X-B, Huang D-H, Song S, Chen Y-J, Tang J, Tao D, Yin Z-N, Mor G and Liao A-H (2021). Front. Immunol. 12:657552. doi: 10.3389/fimmu.2021.657552

Due to a production error, additional proof corrections submitted by the author were not implemented in the final version. Corrections have therefore been made throughout the article.

There was an error in Table 1, Table 2, Figure 1, and Figure 3. Instead of "Characteristics of 12 immune parameters with significant differences in NPW, NP and RPL groups", Table $\mathbf{1}$ should say "Characteristics of 11 immune parameters with significant differences in NPW, NP and RPL

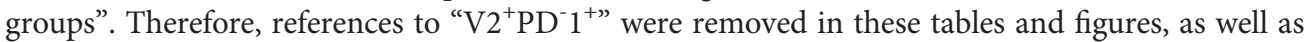
throughout the text, including the Abstract, Introduction and Results.

The corrected tables and figures appear below.

Additionally, Supplementary Figure 1, Supplementary Figure 3, and Supplementary Table 2 were not the latest versions. The correct versions appear below.

The publisher apologizes for these mistakes. The original version of this article has been updated. 
TABLE 1 | Characteristics of 11 immune parameters with significant differences in NPW, NP and RPL groups.

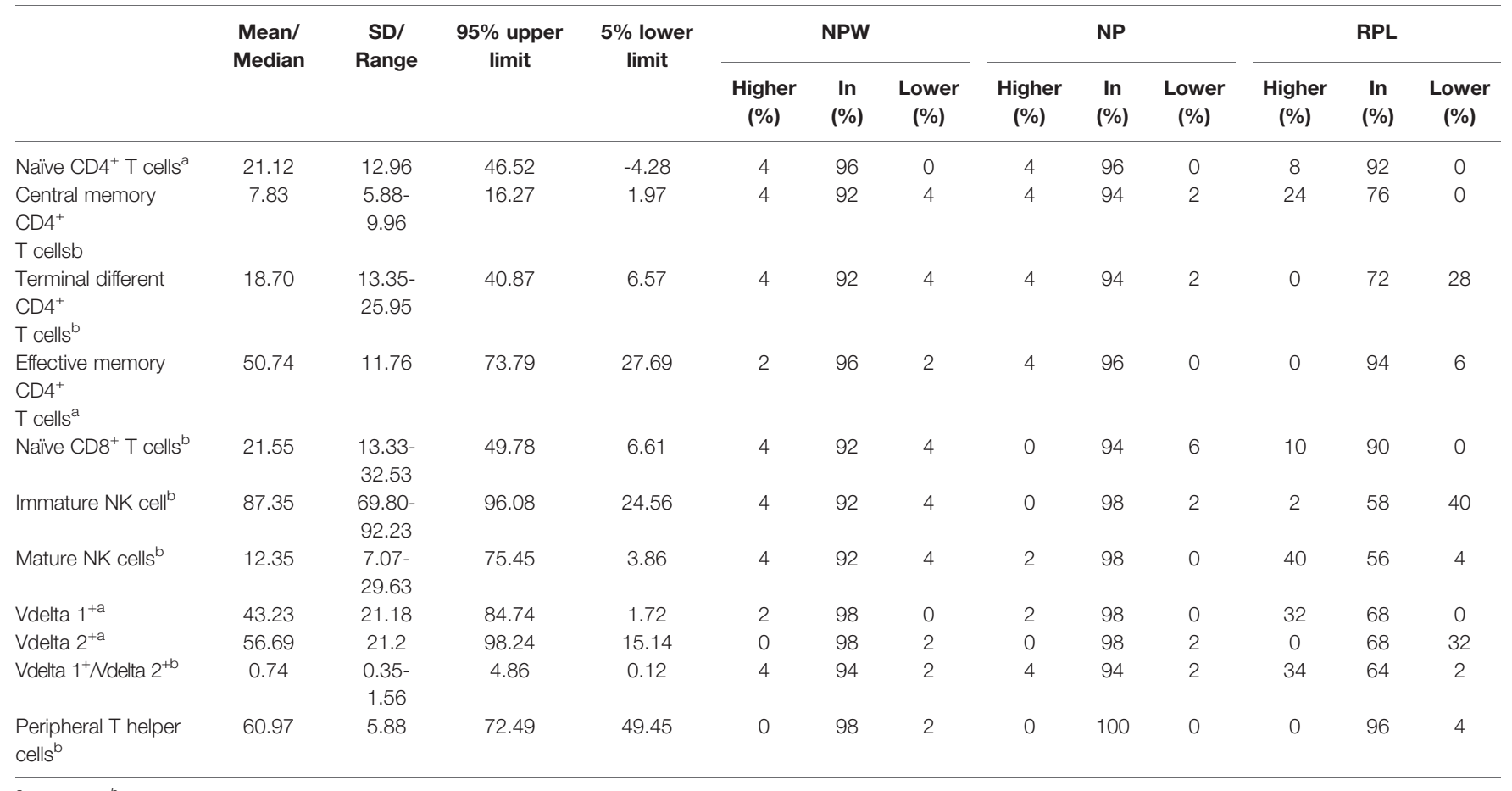

${ }^{a}$ mean/SD; ${ }^{b}$ median/range.

TABLE 2 | Potential functions of significantly different immune cell subsets.

Cell subsets

Potential functions

Ref

Naiive $\mathrm{CD} 4^{+} \mathrm{T}$ cells

Naïve CD8 ${ }^{+} T$ cells

Terminal differentiated CD4 ${ }^{+}$

$\mathrm{T}$ cells

Central memory CD4 ${ }^{+}$

$T$ cells

Effective memory CD4 ${ }^{+}$

$\mathrm{T}$ cells

Immature NK cells

Mature NK cells

$\mathrm{V} \delta 1^{+} \mathrm{T}$ cells

$\mathrm{V} \delta 2^{+} \mathrm{T}$ cells

Peripheral $\mathrm{T}$ helper cells

$\left(\mathrm{T}_{\mathrm{PH}}\right)$
Naïve $\mathrm{CD}^{+}{ }^{+} \mathrm{T}$ cells differentiate to effector $\mathrm{T}$ cells and subsequently develop into long-lived memory $\mathrm{T}$ cells.

Naiive CD8 ${ }^{+} T$ cells differentiate to effector $T$ cells and subsequently develop into long-lived memory $T$ cells.

Terminally differentiated $\mathrm{CD} 4^{+} \mathrm{T}$ cells are associated with protection, though they do not have the ability of renewal and differentiation

Central memory $\mathrm{CD}^{+} \mathrm{T}$ cells mediate reactive memory, readily proliferate, and differentiate to effector cells and produce large amounts of IFN- $\gamma$ or IL-4 in response to antigenic stimulation.

Effective memory $\mathrm{CD}^{+} \mathrm{T}$ cells mediate protective memory, display immediate effector function.

$(32,33)$

$(32,33)$

Immature NK cells have low cytotoxicity and high production of cytokines and chemokines, including

M-CSF and GM-CSF.

Mature NK cells are functionally well known for their potent cytotoxic activity

(37-39)

$V \delta 1^{+} T$ cells possess both regulatory and effector properties. V $\delta 1 \mathrm{~T}$ cells could kill tumor cells and have

$(40-42)$ pro-inflammatory properties.

$\vee \delta 2^{+} \mathrm{T}$ cells exert a cytolytic effect against pathogenic properties.

$\mathrm{T}_{\mathrm{PH}}$ cells are uniquely antigen-specific $\mathrm{T}$ cells with increased expression of genes associated with $\mathrm{B}$ cell 

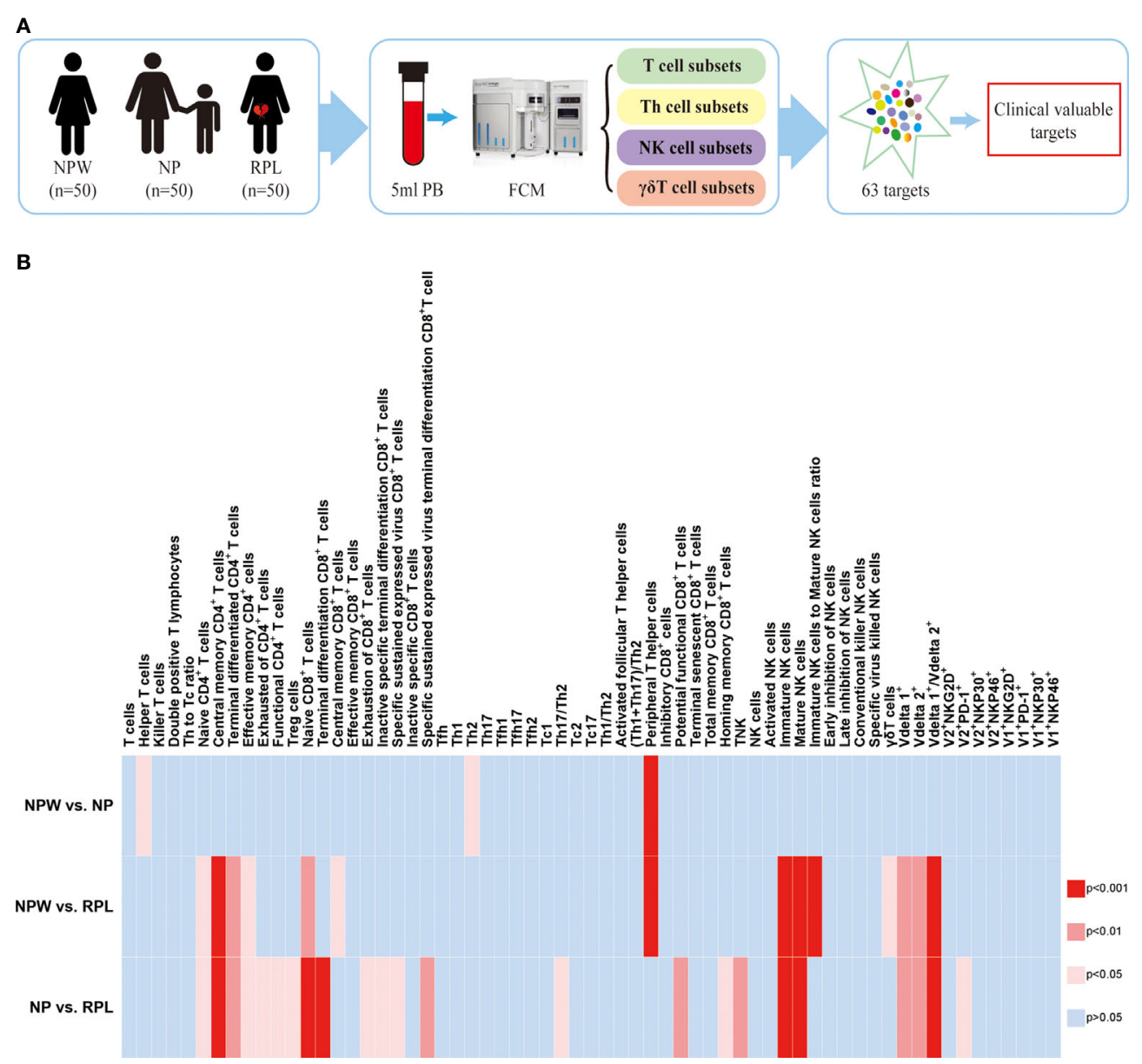

FIGURE 1 | Study design and the overview of the different immunological parameters among the three groups. (A) Schedule of the study. Three groups were included in our study: NPW group (women who were never pregnant, $n=50$ ), NP group (women with the history of normal pregnancy, $n=50$ ) and RPL group (women with the history of RPL, $n=50$ ). Peripheral blood mononuclear cells (PBMCs) were isolated from $5 \mathrm{ml}$ peripheral blood. Total 63 immune cell subsets were simultaneously detected by flow cytometry, including T cell, NK cell and gd T cell subsets. By analyzing the data, clinical-relevant immune parameters were finally identified. (B) Heat map of the significantly changed immune parameters. The 63 immunological parameters were compared among the three groups and presented as the heat map, which can directly show the differences. The colors represent the different significance among the comparisons. The deeper the color is red, the larger the differences are. The blue represents no significance. NPW, women never pregnant; NP, women with a history of normal pregnancy; RPL, women with ahistory of RPL. 

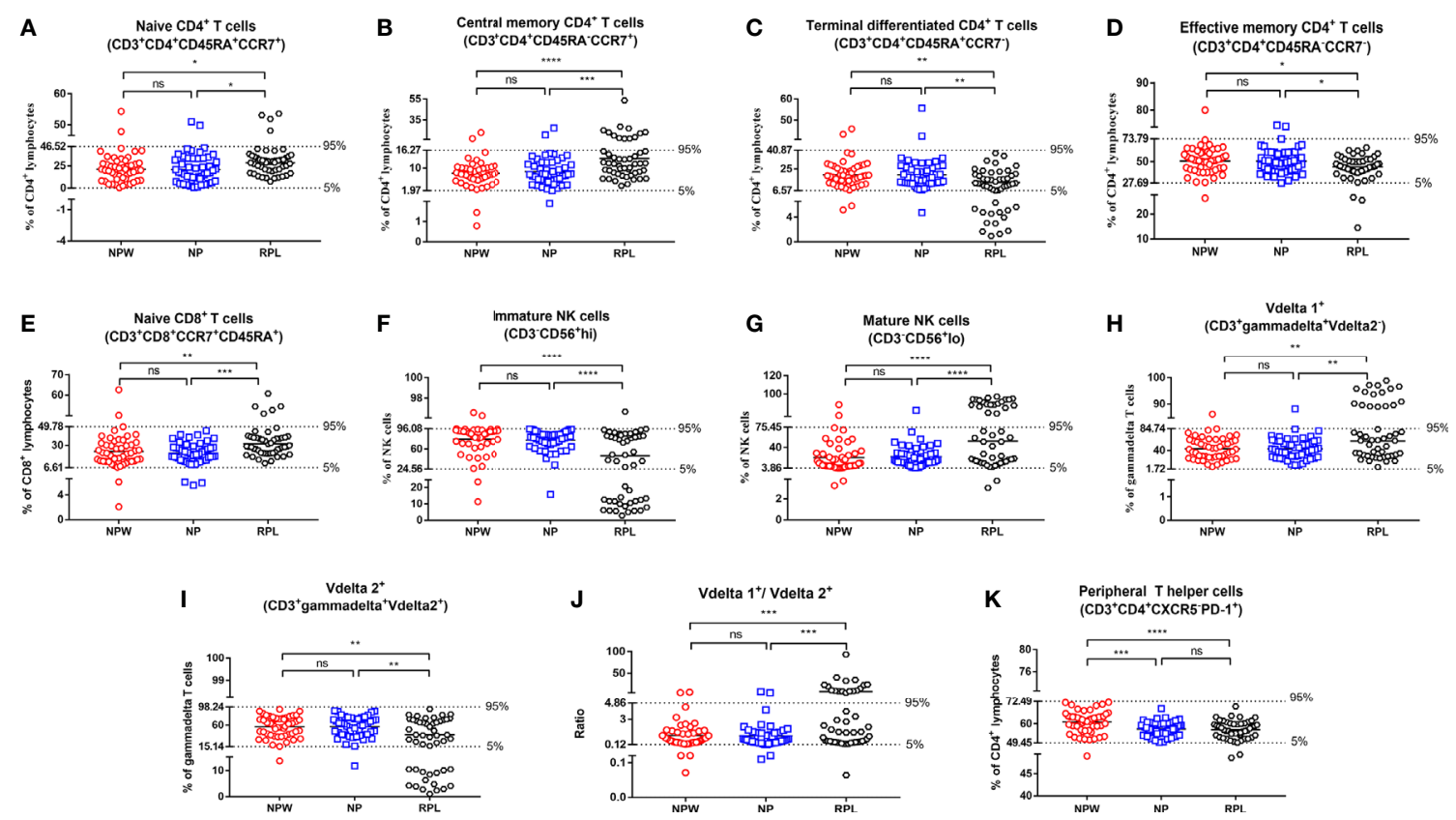

FIGURE 3 | Reference ranges for the significantly changed immune parameters. (A-J) Reference ranges for significantly changed immune parameters related to RPL. The percentages of naiive CD4 $4^{+} T$ cells $(\mathbf{A})$, central memory $C D 4^{+} T$ cells $(\mathbf{B})$, naive $C D 8^{+} T$ cells $(\mathbf{E})$, mature NK cells $(\mathbf{G}), V \delta 1^{+} T$ cells $(\mathbf{H})$ and the ratio of $V \delta 1^{+}$ $T$ cells $/ N \delta 2^{+} T$ cells $(\mathbf{J})$ were significantly higher in the RPL group than those in the NPW and NP groups. The percentages of terminal differentiated CD4 ${ }^{+} T$ cells $(\mathbf{D})$

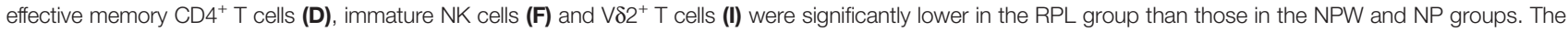
mean ${ }^{+} 1.96 \mathrm{SD}$ was used to measure the reference ranges for the normally distributed data. Median and 5th/95th percentiles represented the lower/upper limit to set up the reference ranges for skewed distribution data. In 14\%- 40\% of women with RPL, the percentages of central memory CD4 ${ }^{+} \mathrm{T}$ cells (B), mature NK cells (G), the ratio of $V \delta 1^{+} T$ cells $/ V \delta 2^{+} T$ cells $(\mathbf{J})$, and $V \delta 1^{+} T$ cells $(\mathbf{H})$ were above the 95th percentile limit. In $28 \%$ - 40\% of women with $R P L$ group, the percentages of terminally differentiated $\mathrm{CD}^{+} \mathrm{T}$ cells $\mathbf{( C )}$, immature NK cells $\mathbf{( F )}$ and $V \delta 2^{+} \mathrm{T}$ cells $\mathbf{( I )}$ were below the 5 th percentile limit. The percentages of these different immunological parameters in the NP group were similar to those in the NPW group, and most were within the 5th percentile limit and 95th percentile limit. (K) Reference ranges for significantly changed immune parameters related to pregnancies. The percentage of TPH was significantly low in the NP and RPL groups

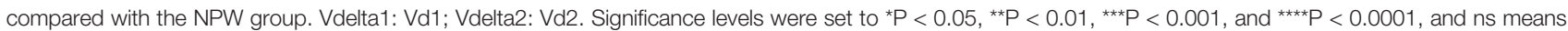
not significant. 


\section{SUPPLEMENTARY MATERIAL}

The Supplementary Material for this article can be found online at: https://www.frontiersin.org/articles/10.3389/fimmu.2021.722805/ full\#supplementary-material

Supplementary Figure 1 | Reference ranges for nonsignificant immune parameters in CD4+ and CD8+ T cells. (A) Th1 and Th17 cells were not significantly different in the RPL vs. NP and RPL vs. NPW groups. Th2 cells were significantly different in the NP vs. NPW groups but not in the RPL vs. NP and RPL vs. NPW groups. Treg cells were significantly different in the RPL vs. NP groups but not in the RPL vs. NPW groups. (B) T cells (a), killer T cells (b), effective memory CD8+ T cells (e), inactive specific CD8+ T cells (i), inhibitory CD8+ T cells ( $k$ ), terminally senescent CD8+ T cells $(m)$ and total memory CD8+ $T$ cells $(n)$ were not significantly different among the three groups. Some of the parameters were lower or higher than the 5 th/ 95th percentile limits. Terminal differentiation CD8+ T cells (c), exhaustion of CD8+ T cells $(f)$, inactive specific terminal differentiation CD8+ T cells $(g)$, specific sustained expressed virus CD8+ T cells (h), specific sustained expressed virus terminal differentiation CD8+ T cells (j), potential functional CD8+ T cells (l), and homing memory CD8+ T cells (o) were significantly different in the RPL vs. NP groups but not in the RPL vs. NPW groups. Central memory CD8+ T cells (d) were significantly different in the NPW vs. RPL groups but not in RPL vs. NP groups. (C) CD4+ T cell DP T lymphocytes and activated Tfh cells were not significantly different among the

\section{REFERENCES}

32. Sprent J, Surh CD. Normal T Cell Homeostasis: The Conversion of Naive Cells Into Memory-Phenotype Cells. Nat Immunol (2011) 12:478-84. doi: 10.1038/ni.2018

33. Weiskopf D, Bangs DJ, Sidney J, Kolla RV, De Silva AD, de Silva AM, et al. Dengue Virus Infection Elicits Highly Polarized CX3CR1+ Cytotoxic CD4+ T Cells Associated with Protective Immunity. Proc Natl Acad Sci USA (2015) 112:E4256-63. doi: 10.1073/pnas.1505956112

34. Crotty S. Follicular Helper CD4 T Cells (TFH). Annu Rev Immunol (2011) 29:621-63. doi: 10.1146/annurev-immunol-031210-101400

35. Cai D, Tang Y, Yao X. Changes of GammadeltaT Cell Subtypes During Pregnancy and Their Influences in Spontaneous Abortion. J Reprod Immunol (2019) 131:57-62. doi: 10.1016/j.jri.2019.01.003

36. Uchida Y, Gherardini J, Schulte-Mecklenbeck A, Alam M, Cheret J, Rossi A, et al. Pro-Inflammatory Vdeltal(+)T-Cells infiltrates Are Present in and Around the Hair Bulbs of Non-Lesional and Lesional Alopecia Areata Hair Follicles. J Dermatol Sci (2020) 100:129-38. doi: 10.1016/j.jdermsci.2020.09.001

37. Brummelman J, Pilipow K, Lugli E. The Single-Cell Phenotypic Identity of Human CD8(+) and CD4(+) T Cells. Int Rev Cell Mol Biol (2018) 341:63124. doi: $10.1016 /$ bs.ircmb.2018.05.007

38. Gordon CL, Miron M, Thome JJ, Matsuoka N, Weiner J, Rak MA, et al. Tissue Reservoirs of Antiviral T Cell Immunity in Persistent Human CMV Infection. J Exp Med (2017) 214:651-67. doi: 10.1084/jem.20160758

39. Gattinoni L, Lugli E, Ji Y, Pos Z, Paulos CM, Quigley MF, et al. A Human Memory T Cell Subset With Stem Cell-Like Properties. Nat Med (2011) 17:1290-7. doi: $10.1038 / \mathrm{nm} .2446$ three groups. Helper T cells were significantly different in the NP vs. NPW groups. The exhaustion of CD4+ T cells and functional CD4+ T cells was significant in the RPL vs. NP groups but not in the RPL vs. NPW groups. (D) Tfh cells (a) and cytotoxic T (Tc) cells (Tfh, Tfh1, Tfh2, Tfh17, Tc1, Tc2 and Tc17) (b-g) were not significantly different among the three groups. (E) The Th to Tc ratios (a), Th1/Th2 ratios (c) and (Th1+Th17)/Th2 ratios (d) were nonsense parameters among the three groups. Th17/Th2 (b) was significantly different in the RPL vs. NP groups. The ratio of immature NK cells to mature NK cells (e) was significantly different in the RPL vs. NPW groups. The mean $\pm 1.96 \mathrm{SD}$ was used to measure the reference range for the normally distributed data. The median and 5th/95th percentiles represent the lower/upper limits of each reference range for skewed distribution data. Significance levels were set to ${ }^{*} P<0.05,{ }^{\star \star} P<0.01$, and ${ }^{\star \star \star} P<0.001$ and ns means not significant.

Supplementary Figure 3 | Reference ranges for nonsignificant immune parameters in gd T cells. (A) $\gamma \delta$ T cells were significantly different in the RPL vs. NPW groups. (B-I) The percentages of $\mathrm{V} 1+\mathrm{NKG} 2 \mathrm{D}+, \mathrm{V} 1+\mathrm{NKP} 30+, \mathrm{V} 1+\mathrm{NKP} 46+, \mathrm{V} 1$ $+\mathrm{PD}-1+, \mathrm{V} 2+\mathrm{NKG} 2 \mathrm{D}+, \mathrm{V} 2+\mathrm{NKP} 30+$ and $\mathrm{V} 2+\mathrm{NKP} 46+\gamma \delta \mathrm{T}$ cells were not significantly different among the three groups. The percentage of Vdelta2+PD- $1+\gamma \delta$ $T$ cells was significantly different between the RPL and NP groups $(P<0.05)$. (I) The mean $\pm 1.96 \mathrm{SD}$ was used to measure the reference range for the normally distributed data. The median and 5th/95th percentiles represent the lower/upper limits of the reference range for skewed distribution data. Significance levels were set to $\mathrm{P}<0.05\left({ }^{*}\right)$, and $n s$ means not significant.

40. Peters C, Kabelitz D, Wesch D. Regulatory Functions of Gammadelta T Cells. Cell Mol Life Sci (2018) 75:2125-35. doi: 10.1007/s00018-018-2788-x

41. Xu B, Pizarro JC, Holmes MA, McBeth C, Groh V, Spies T, et al. Crystal Structure of A Gammadelta T-Cell Receptor Specific for the Human MHC Class I Homolog MICA. Proc Natl Acad Sci USA (2011) 108:2414-9. doi: 10.1073/pnas.1015433108

42. Simoes AE, Di Lorenzo B, Silva-Santos B. Molecular Determinants of Target Cell Recognition by Human Gammadelta T Cells. Front Immunol (2018) 9:929. doi: 10.3389/fimmu.2018.00929

43. Ribeiro ST, Ribot JC, Silva-Santos B. Five Layers of Receptor Signaling in gammadelta T-Cell Differentiation and Activation. Front Immunol (2015) 6:15. doi: 10.3389/fimmu.2015.00015

44. Chen Y, Mo J, Jia X, He Y. The B7 Family Member B7-H6: A New Bane of Tumor. Pathol Oncol Res (2018) 24:717-21. doi: 10.1007/s12253-0170357-5

45. Cantoni C, Bottino C, Vitale M, Pessino A, Augugliaro R, Malaspina A, et al. NKp44, A Triggering Receptor Involved in Tumor Cell Lysis by Activated Human Natural Killer Cells, Is a Novel Member of the Immunoglobulin Superfamily. J Exp Med (1999) 189:787-96. doi: 10.1084/jem.189.5.787

Copyright $\odot 2021$ Frontiers Production Office. This is an open-access article distributed under the terms of the Creative Commons Attribution License (CC BY). The use, distribution or reproduction in other forums is permitted, provided the original author(s) and the copyright owner(s) are credited and that the original publication in this journal is cited, in accordance with accepted academic practice. No use, distribution or reproduction is permitted which does not comply with these terms. 